\title{
Sacubitril/Valsartan Conundrum
}

\author{
John Somberga, b
}

Sacubitril/valsartan (Entresto ${ }^{\circledR}$ ) has been touted as the new wonder drug for heart failure (HF). Sacubitril is a prodrug that is activated in man to sacubitrilat that inhibits the enzyme neprilysin, a neutral endopeptidase. This enzyme degrades vasomotor peptides, most importantly natriuretic peptides: bradykinin and adrenomedullin. Sacubitril causes an increase in angiotensin II and thus needs an angiotensin II receptor blocker (ARB) to be combined with it to prevent excessive vasoconstriction. By inhibiting neprilysin (sacubitril effect) and by blocking angiotensin II (valsartan action) one has a very potent vasodilator action.

There is a potential downside in inhibiting neprilysin since neprilysin increases the clearance of amyloid beta, a substance whose buildup in the brain is thought a cause of Alzheimer's disease alibi over a very long time period. Whether this is a concern or not is currently unknown, with no data supporting adversity though exposure times have been far too short to make a determination.

The drug combination was approved and vaulted to the top of guideline recommendations on the basis of a single study PARADIGM-HF. This was a large (8,442 patients) study over 27 months looking at the incidence of first hospitalization or death. The study found $27 \%$ reduction in the positive endpoint in the control group (standard therapy) versus $22 \%$ reduction in the sacubitril/valsartan group with a reported $\mathrm{P}$ value of $<$ 0.001 . Only $1 \%$ of the patients had severe HF, New York Heart Association (NYHA) class IV, and the patients by virtue of study entry criteria were quite stable. With a single study some researchers and clinicians were skeptical. However, the consensus view was that with a $\mathrm{P}<0.001$ the results were "the same as if two independent studies were performed and found $\mathrm{P}<0.05$ significance" for each of the two theoretical studies.

This reasoning often stated is not correct. Each independent study has its individual characteristics. A study population is unique and at times cannot be generalized to the entire population to be treated. Randomization may have its quirks and unknown biases that may have entered into patient selection, study methods and data analysis. Thus, two separate studies are different and more meaningful than one highly significant study as determined by a $\mathrm{P}$ value. This point of view may be

Manuscript submitted May 8, 2021, accepted May 10, 2021

Published online May 14, 2021

aCardiology \& Pharmacology, Rush University, Chicago, IL 60612, USA.

Email: John_Somberg@rush.edu

bEditor-in-Chief, Cardiology Research

doi: https://doi.org/10.14740/cr1278 just viewed as theatrical, but in light of subsequent findings may have merit.

A second study with sacubitril/valsartan was undertaken in patients with more severe HF but was unfortunately stopped at 24 weeks due to coronavirus disease 2019 (COVID-19) epidemic, and is not determinative. The recent report of the PARADISE-MI study failing to show a significant benefit is even more troubling. The basic tenant of an angiotensin-converting enzyme (ACE) inhibitor and ARBs is that they inhibit angiotensin II action, thus reducing blood pressure (BP) and impede remodeling. This should occur in HF and post myocardial infarction (MI). That a neprilysin inhibitor offers no benefit post MI over an ACE or ARB is perplexing. Was the PARAGON-HF study an outlier, showing far more benefit than actually exists? This is speculation, but speculation based on the incomplete assessment of sacubitril/valsartan and the failure of PARADISE-MI. Perhaps a more thorough evaluation of sacubitril/valsartan is needed before its position as guidelinedirected optimum HF therapy is accepted.

\section{Acknowledgments}

None to declare.

\section{Financial Disclosure}

None to declare.

\section{Conflict of Interest}

None to declare.

\section{Data Availability}

The author declares that data supporting the findings of this study are available within the article. 